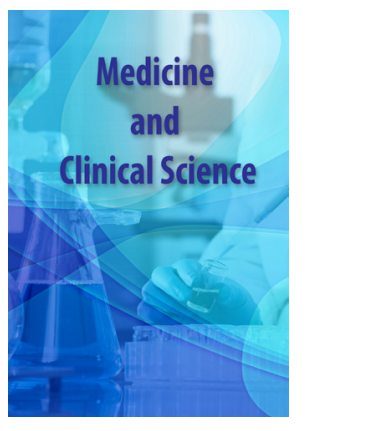

Correspondence

Edafe EA

Bayelsa Specialist Hospital, Yenagoa, Nigeria E-mail: dremmanueledafe@gmail.com

- Received Date: 22 Dec 2019;

- Accepted Date: 14 Jan 2020;

- Publication Date: 18 Jan 2020

Keywords

Coronary Artery Disease, Diabetes Mellitus, Pattern of Dyslipidemia, Hypertension

\section{Pattern of dyslipidaemia in coronary artery disease in south-South Nigeria}

\author{
Edafe EA ${ }^{1 *}$ and Dodiyi-Manuel $\mathrm{ST}^{2}$ \\ 'Bayelsa Specialist Hospital, Yenagoa, Nigeria \\ 2University of Port Harcourt Teaching Hospital, Port Harcourt, Nigeria
}

Copyright

(c) 2020 Science Excel. This is an openaccess article distributed under the term of the Creative Commons Attribution 4.0 International license.

\begin{abstract}
Introduction: Dyslipidemia is an independent traditional risk factor for coronary artery disease (CAD). The pattern of dyslipidemia and coronary artery disease among sub-Saharan Africans has not been widely studied. Hence, this study was conducted with the objective to evaluate pattern of lipid abnormalities in patients with established coronary artery disease. Methods: It was a prospective study of the pattern of dyslipidemia among patients with established coronary artery disease from angiographic diagnosis. All patients with dyslipidemia who had coronary angiography were evaluated for pattern of lipid profile. Results: There were a total of 65 subjects with age ranging from 35 to 85 years (Male: 34; Female: 31 ). The mean age of participants was $62.1 \pm 12.0$ years. There were more subjects with mixed (combined) dyslipidemia who had significant coronary artery disease compared with isolated dyslipidemia ( $p$-value $<0.001$ ). There was no significant difference between the male and female with dyslipidemia. The mean values of total cholesterol, low density lipoprotein and triglycerides were higher among the subjects with dyslipidemia. Conclusion: The study showed that combined dyslipidemia is commonly seen in patients with coronary artery disease as compared with isolated dyslipidemia.
\end{abstract}

\section{Introduction}

Cardiovascular disease (CVD) is a major health problem across the world, accounting for $30 \%$ of all deaths [1]. CVD is on the increase, especially in developing countries. This may be due to a combination of demographic and epidemiologic transitions of those living in developing countries [2].

Dyslipidaemia is a key factor in the pathophysiology of CVDs [3]. It is one of the four major established conventional modifiable risk factors for coronary artery disease. The others include cigarette smoking, diabetes mellitus, and hypertension [4]. Dyslipidaemia is a metabolic abnormality that results in persistent increase in the plasma concentration of cholesterol and triglycerides. It may be classified into the following types: isolated dyslipidaemia (any one component of lipid profile is abnormal); mixed (combined) dyslipidaemia (at least two components of lipid profile are abnormal [5].

The pattern of dyslipidemia among patients with coronary artery disease has not been fully explored among Nigerians in the sub-Saharan African. Hence, the objective of the study was to evaluate the lipid profile with regard to pattern of dyslipidaemia among patients that were investigated for coronary artery disease $(\mathrm{CAD})$ at the Bayelsa Specialist Hospital, Yenagoa in south-south Nigeria.

\section{Methods}

This was a prospective descriptive study conducted between 1st October 2017 and 31st April 2019. The subjects included 65 adults that were referred to the cardiac catheterization laboratory of Bayelsa Specialists Hospital, Yenagoa. The subjects were recruited by convenience sampling. The center serves as a referral Cardiology facility for the South-East and South-South parts of Nigeria.

The participants were investigated for coronary artery disease, following presentation with chest pain, dizziness, palpitations, etc. The lipid profile was carried out for all included subjects. Patients already on lipid lowering medications or had a past history of dyslipidemia were excluded from the study.

The lipid profile was done after overnightfast for at least 8 hours prior to collection of samples. The lipid profile components investigated were total cholesterol (TC), triglycerides (TG), low density lipoprotein - C (LDL-C), and high-density lipoprotein - C (HDL-C)) [6]. Values of TC $\geq 200 \mathrm{mg} / \mathrm{dL}$ were considered significant for hypercholesterolaemia, TG $\geq 150 \mathrm{mg} /$ $\mathrm{dL}$ for hypertriglyceridaemia, LDL-C $\geq 130 \mathrm{mg} / \mathrm{dL}$ for LDL-C hyperlipidaemia, while HDL-C $\leq 40 \mathrm{mg} /$ $\mathrm{dL}$ in males and $\leq 50 \mathrm{mg} / \mathrm{dL}$ in females for HDL-C dyslipidaemia. Mixed(Combined) dyslipidaemia includes abnormality or derangement of at least two components of the lipid profile. 


\section{Definitions of terms}

Dyslipidemia was diagnosed according to the criteria set by the National Cholesterol Education Program-Adult Treatment Panel III (NCEP-ATP III) and classified into four phenotypes [11]. Isolated dyslipidemia is abnormality in one of the parameters of lipid profile. (a) isolated hypertriglyceridemia was defined as having serum triglycerides $\geq 150 \mathrm{mg} / \mathrm{dl}$ and total cholesterol $\leq 200 \mathrm{mg} / \mathrm{dl}$; (b) isolated hypercholesterolemia was defined as having total cholesterol $\geq 200 \mathrm{mg} /$ $\mathrm{dl}$ and triglycerides $\leq 150 \mathrm{mg} / \mathrm{dl}$; (c) mixed (combined) hyperlipidemia was defined as having triglycerides $\geq 150 \mathrm{mg} / \mathrm{dl}$ and total cholesterol $\geq 200 \mathrm{mg} / \mathrm{dl}$; and (d) isolated low HDL-C was defined as having HDL-C $\leq 40 \mathrm{mg} / \mathrm{dl}$ in male and $\leq 50 \mathrm{mg} / \mathrm{dl}$ in female without hypercholesterolemia nor hypertriglyceridemia [12]. Mixed (combined) dyslipidemia is one in which there is an elevated LDL cholesterol and triglyceride levels combined with decreased levels of HDL cholesterol. This form of dyslipidemia is commonly seen in patients with diabetes and metabolic syndrome.(13)

Smoking: An active smoker was defined as a person who currently smoked at least one cigarette a day. An ex-smoker was defined as a person who had regularly smoked but had not smoked in the previous month. Second-hand smoking was a non-smoker who had regular exposure to tobacco products.

Diabetes mellitus was defined as patients on anti-diabetic medications or FBG $\geq 7.0 \mathrm{mmol} / \mathrm{l}$ or $\mathrm{HbAlc} \geq 6.5 \%$. Hypertension was defined as a subject on anti-hypertensive medications or a persistent BP of $\geq 140 / 90$ $\mathrm{mmHg}$ at least two different measurement. Dyslipidemia was defined as the presence of any abnormalities in the lipid profile, occurring alone or in combination. Sedentary life style is an adoption of habits known to promote cardiovascular diseases.

\begin{tabular}{|c|c|c|c|c|}
\hline Variable & Category & Frequency & Percentage & p-Value \\
\hline \multirow{6}{*}{$\begin{array}{l}\text { Age } \\
\text { (years) }\end{array}$} & $<40$ & 3 & 4.6 & \\
\hline & $40-49$ & 18 & 27.7 & \\
\hline & $50-59$ & 11 & 16.9 & \\
\hline & $60-69$ & 21 & 32.3 & \\
\hline & $70-79$ & 7 & 10.8 & \\
\hline & $\geq 80$ & 5 & 7.7 & 0.096 \\
\hline \multirow{3}{*}{ Education } & Primary & 22 & 33.8 & \\
\hline & Secondary & 20 & 30.8 & \\
\hline & Tertiary & 23 & 35.4 & 0.681 \\
\hline \multirow{7}{*}{ occupation } & legislative & 1 & 1.5 & \\
\hline & Executive & 14 & 21.5 & \\
\hline & $\begin{array}{c}\text { Business } \\
\text { owners }\end{array}$ & 15 & 23.1 & \\
\hline & $\begin{array}{l}\text { Senior civil } \\
\text { servants }\end{array}$ & 12 & 18.5 & \\
\hline & $\begin{array}{l}\text { Junior civil } \\
\text { servants }\end{array}$ & 9 & 13.8 & \\
\hline & Pretty traders & 9 & 13.8 & \\
\hline & Others & 5 & 5.7 & 0.896 \\
\hline
\end{tabular}

Table 1. Demographic profile of subjects

\section{Statistical analysis}

Statistical analysis was performed using the 20th version of Statistical Package for Social Sciences (SPSS), IBM cooperation 2011, Armonk, New York, USA. The results were presented in tabular or graphical forms, as were appropriate. All the continuous variables (e.g. age, TC, TG, LDL-c, and HDL-c values) were subjected to a normality testing using Q-Q plots and were found to be normally distributed; hence the parametric tests were subsequently used for analysis.

Age was grouped as $<40,40-49,50-59,60-69,70-79$ and $\geq 80$ for the patients, and were compared using chi-square test of significance for difference in proportion. Gender, educational status, and occupation were also compared for subjects and controls using chi-square test of significance. Laboratory characteristics of patients (TC, TG, HDL-c, LDL-c) were compared for difference in respective means using independent sample t-test, and presented as appropriate.

\section{Results}

The baseline characteristics of the study participants are shown in Table 1 . The study population consisted of 65 participants with ages ranging from 35 to 85 years. The average age of the participants was $62.1 \pm 8.0$. There were 34 males and 31 females. The mean age of men in the study was $63.4 \pm 9.4$ years while that of women was $61.7 \pm 10.1$ years. There was no stastically significant difference between both sex $(\mathrm{p}=0.462)$.

All the patients had received at least primary level of education. Out of the 65 patients referred to the cardiovascular center 50(76.9\%) had significant CAD. Twenty seven $(54.0 \%)$ were males, while $23(46.0 \%)$ females (Figure1).

There was no statistically difference in the aged grouped, level of education and the occupation as shown in Table 1.
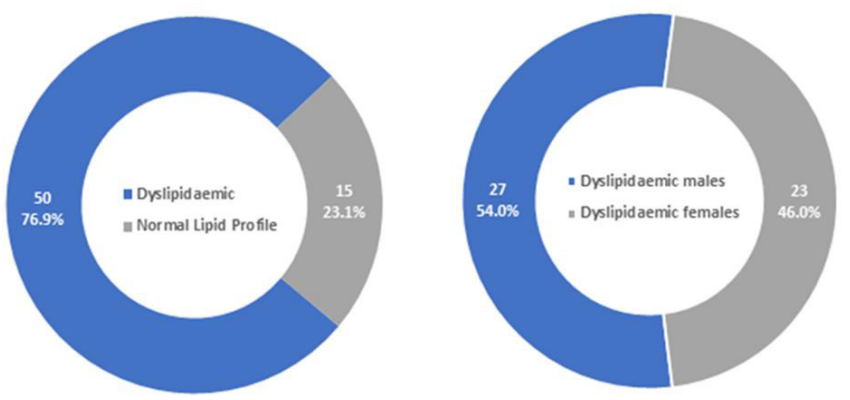

Figure 1. 1a: showed that 50 subjects had abnormal lipid profile; and 15 had nomal lipid profile. $1 \mathrm{~b}$ : showed that 27 of the 50 with dyslipidemia were male.

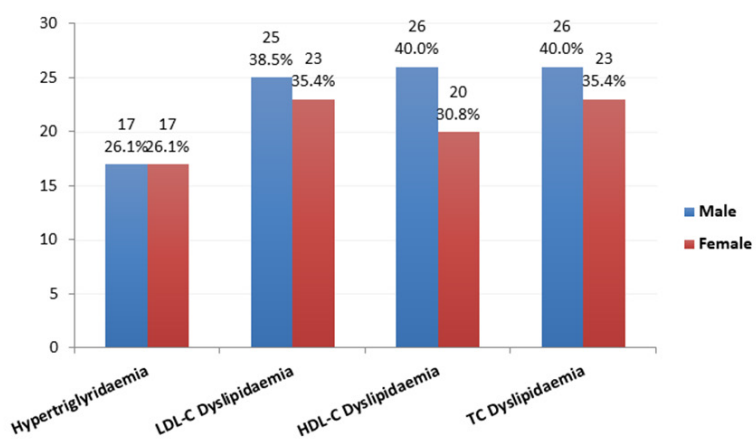

Figure 2. Pattern of dyslipidaemia among patients investigated for CAD 


\begin{tabular}{|l|c|c|c|c|}
\hline Characteristics & Total $(\mathbf{n}=\mathbf{6 5})$ & Male $(\mathbf{n}=\mathbf{3 4})$ & Female $(\mathbf{n}=\mathbf{3 1})$ & $\mathbf{p}-$ Value \\
\hline TC $(\mathrm{mg} / \mathrm{dL})$ & $247.4 \pm 82.9$ & $232.1 \pm 70.7$ & $262.8 \pm 94.2$ & 0.14 \\
\hline HDL-C (mg/dL) & $46.3 \pm 8.7$ & $45.3 \pm 8.2$ & $47.5 \pm 9.4$ & 0.32 \\
\hline LDL-C (mg/dL) & $143.1 \pm 36.4$ & $140.8 \pm 28.7$ & $145.1 \pm 44.3$ & 0.64 \\
\hline TG (mg/dL) & $157.3 \pm 66.3$ & $142.9 \pm 46.6$ & $170.6 \pm 80.7$ & 0.11 \\
\hline LDL-C hypercolestrolaemia & 47.00 & $25(73.5)$ & $22(71.0)$ & 0.99 \\
\hline TC hypercolestrolaemia & 48.00 & $26(76.5)$ & $22(71.0)$ & 0.77 \\
\hline HDL-C hypercolestrolaemia & 45.00 & $26(76.5)$ & $19(31.3)$ & 0.25 \\
\hline TG hypercolestrolaemia & 33.00 & $17(50.0)$ & $16(51.6)$ & 0.79 \\
\hline Isolated Dyslipidemia & 3.00 & $1(2.9)$ & $2(6.5)$ & 0.09 \\
\hline Combined (Mixed) Dyslipidemia & 47.00 & $26(76.5)$ & $21(67.7)$ & 0.43 \\
\hline Dyslipidemia & 50.00 & $27(79.4)$ & $23(74.2)$ & 0.39 \\
\hline
\end{tabular}

Table 2. The serum lipid concentrations and dyslipidemia patterns in both genders

\begin{tabular}{|l|c|c|c|c|c|}
\hline Lipid Profile Component & Minimum Statistic & Maximum Statistic & Mean Statistic & Std. Error & Deviation Statistic \\
\hline TC (mg/dL) & 85.00 & 465.00 & 247.03 & 10.30 & 82.75 \\
\hline TG (mg/dL) & 69.00 & 340.00 & 155.88 & 8.10 & 65.27 \\
\hline LDL-C hypercolestrolaemia & 69.00 & 303.00 & 142.78 & 4.50 & 36.33 \\
\hline HDL-C hypercolestrolaemia & & & & & \\
\hline Male & 34.00 & 62.00 & 45.31 & 1.08 & \\
\hline Female & 34.00 & 64.00 & 47.45 & 1.62 & 9.21 \\
\hline TC: Total cholestrol; TG: Triglyceride; LDL-C: Low-Density Lipoprotien-C; HDL-C: High-Density Lipoprotien-C; \\
\hline
\end{tabular}

Table 3. Lipid profile of CAD Patients.

The risk factors among these participants were diabetes mellitus, hypertension, smoking and sedentary life style. Thirty two were diabetic, 21 were hypertensive, 15 with history of active smoking and 46 with sedentary life style.

As shown in Table 2, total dyslipidemia, isolated hypercholesterolemia and low HDL-C in women were not significantly higher women than men. There was also no statistically significant difference for isolated hypertriglyceridemia and mixed hyperlipidemia between both genders.

The descriptive statistics of the lipid profile of the participants investigated for CAD were shown in Table 2. The mean HDL-C values were reduced in both participants. Other lipid profile parameters mean values were above upper limit for normo-lipidaemic subjects.

Fifty subjects were found with dyslipidemia among the 65 participants. Forty seven and 2 participants had combined and isolated dyslipidemia respectively. Male subjects (54\%) with dyslipidemia were slightly higher than their female counterparts (46\%). This was not statistically significant with a $p$-value $=0.285$. This is shown in in figure 1 .

The pattern of dyslipidaemia among patients referred to the center for evaluation of CAD following complaints of chest pain is shown in figure 2. Roughly equal proportions of male had LDL-c, HDL-c, TC, and combined dyslipidaemia respectively. Similarly, roughly equal proportions of female had LDL-C, HDL-C, TC, and combined dyslipidaemias respectively.

The pattern of dyslipidaemia observed among patients diagnosed with CAD at the center during the study period is shown in figure 3. Patients with TG dyslipidaemia were the least proportion (19.7\% and $18.0 \%$ among males and females respectively). The proportions of male and female patients with LDL-C, HDL-C, TC, and combined dyslipidaemia show no statistical significant with p-value of 0.914 .

Figure 4 shows the pattern of dyslipidaemia among patients investigated for CAD at the facility. The majority $(94.0 \%)$ of the patients had combined (mixed) type of dyslipidaemia. There was statistical significant difference among participant with combined and isolated dyslipidemia' with p-value $<0.001$.

\section{Discussion}

The patients in this study had primary, secondary or tertiary level of education. This reflected the good literacy level of the south-south Nigerian population consistent with $90.8 \%$ adult literacy rate in English language for south-south in 2010 National Literacy Survey by the Nigerian National Bureau of Statistics.[10] However, it did not reflect the level of awareness for coronary artery disease among the population. Majority of Nigerians still have the poor knowledge of heart disease and its risk factors even though they have primary, secondary or tertiary level of education [11]. Sedentary life style was a major risk factor among participants that were were business and office workers.

There is increasing incidence of dyslipidemia worldwide. This incidence varied among age groups, sex, race/ethnicity, socioeconomic, and cultural characteristics. Findings from this study show a high incidence of dyslipidaemia among men and women with coronary artery disease. This was similar to these prospective studies that evaluated the impact of dyslipidemia on cardiovascular disease $[12,13]$.

Epidemiological studies have shown that increased LDL cholesterol and non-HDL cholesterol levels and decreased HDL cholesterol levels are 
associated with an increased risk of cardiovascular disease in patients with diabetes [14-17]. It is universally accepted that elevated levels of LDL cholesterol and non-HDL cholesterol cause atherosclerosis and cardiovascular disease [18]. This was in keeping with our prospective study which showed that out of the 65 subjects evaluated for coronary artery disease, 50 subjects had dyslipidemia.

In our study, the major types of dyslipidemia among the south-south Nigerians presenting to the cardiac catheterization laboratory were isolated and combined dyslipidemia. Combined dyslipidemia is commoner among the coronary artery disease with a ratio of 15.7:1. This possibly relates to increase intake of high simple carbohydrates and high-fat diet, which obviously affects the serum triglyceride concentration [19].

An estimate of $30-60 \%$ of subjects with Type 2 diabetes have dyslipidemia $[20,21]$. Subjects with Type 2 diabetes often have an increase in serum triglyceride levels and decreased HDL cholesterol levels. Non-HDL cholesterol levels are increased due to the increase in VLDL and IDL $[20,21]$. LDL cholesterol levels are typically not different than in normal subjects but there is an increase in small dense LDL, a lipoprotein particle that is atherogenic. The result is more LDL particles, which coupled with the increases in VLDL and IDL, leads to an increase in Apo B [22].

Interestingly, there was no significant difference among males and females with dyslipidemia. This was in contrast to the Wu et al who reported that it was higher in men than in women among people under age 50 years, but was reversed among people older than 50 years. [23] The reason may be due to small numbers of the subject recruited into the study and that it was among patients evaluated for chest pain.

Abd alamir at al showed that combined hyperlipidemia and isolated hypercholesterolemia are associated with coronary artery disease with risk of multi-vessels involvement [24]. This was similar to our study.

There were some limitations of this study. First, this was a retrospective cross-sectional study; therefore, the causal associations between the risk factors and dyslipidemia cannot be inferred. Second, the investigated population was those who presented to the hospital for chest pain evaluation and it may not reflect the community population.

\section{Conclusion}

This study showed that combined dyslipidemia is more frequent patients with coronary artery disease as compared with isolated dyslipidemia. Whether this trend is common in other regions of Nigeria need to be evaluated. Hence, there is a need for trials with regards to type of dyslipidemia and its treatment in the sub-Saharan Africa.

\section{Conflict of interest}

Authors declare no conflict of interest

\section{References}

1. Moodie DS. The Global Burden of Cardiovascular Disease. Congenit Heart Dis 2016;11(213)1-29.

2. Oguoma VM, Nwose E, Skinner T, Digban K, Onya I, Richards R. Prevalence of cardiovascular disease risk factors among Nigerian adult population: relationship with income level and accessibility to CVD risks screening. BMC Public Heal 2015;15(397):1-16.

3. Haffnar M. Diiabetes, hyperlipidaemia and coronary artery disease. Am J Cardiol 1999;83:17-21.

4. Verschuren WM, Jacobs DR, Bloemberg BP, Kromhout D, Menotti A, Aravanis $C$, et al. Serum total cholesterol and long term coronary heart disease mortality in different cultures: twenty-five follow-up of the seven countries study. JAMA 1995;274:131-6.

5. Ama-Moor V, Amougou S, Ombotto S, Wouamba D, Nonga B. Dyslipidaemia in patients with a cardiovascular risk and disease at the
University Teaching Hospital of Yaounde. Int J Vasc Med 2017;5:1-6.

6. The IDF consensus worldwide definition of the metabolic syndrome. 2006.1-24.

7. Marchesini G, Forlani G, Cerrelli F, Manini R, Natale S, Baraldi L, et al. WHO and APTIII proposals for the definition of the metabolic syndrome in patients with type 2 diabetes. Diabetic Med. 2004;21:383-7.

8. Fan JG, Peng YD. Metabolic syndrome and non-alcoholic fatty liver disease: Asian definitions and Asian studies. Hepatob Pancreat Dis Int. 2007; 6:572-8.

9. Panikar V. Mixed dyslipidemia in Medicine update 2008;18:764-9.

10. National literacy survey, editors. National bureau of statistics. 2010.p. 22. www .nigerian.stat.gov.ng. (accessed, October 25th, 2019).

11. Akintunde AA, AkintundeTS, Opadijo OG. Knowledge of heart disease risk factors among workers in a Nigerian University: A call for concern. Niger Med J 2015;56:91-5.

12. Joshi P, Islam S, Pais P, Reddy S, Dorairaj P, Kazmi K, et al. Risk factors for early myocardial infarction in South Asians compared with individuals in other countries. JAMA. 2007;297:286-94.

13. Enas EA, Mehta J. Malignant coronary artery disease in young Asian Indians: Thoughts on pathogenesis, prevention, and therapy. Coronary Artery Disease in Asian Indians Study. Clin Cardiol. 1995;18:131-5.

14. Feingold KR, Siperstein MD. Diabetic vascular disease. Adv Intern Med. 1986;31:309-40.

15. Fox CS, Golden SH, Anderson C, Bray GA, Burke LE, de Boer IH, et al. Update on Prevention of Cardiovascular Disease in Adults With Type 2 Diabetes Mellitus in Light of Recent Evidence: A Scientific Statement From the American Heart Association and the American Diabetes Association. Diabetes Care. 2015;38(9):1777-803

16. de Ferranti SD, de Boer IH, Fonseca V, Fox CS, Golden SH, Lavie CJ, et al. Type 1 diabetes mellitus and cardiovascular disease: a scientific statement from the American Heart Association and American Diabetes Association. Circulation. 2014;130(13):1110-30.

17. Martín-Timón I, Sevillano-Collantes C, Segura-Galindo A, Del CañizoGómez FJ. Type 2 diabetes and cardiovascular disease: Have all risk factors the same strength? World J Diabetes. 2014;5(4):444-70.

18. Hovingh GK, Rader DJ, Hegele RA. HDLc re-examined. Curr Opin Lipidol. 2015;26(2):127-32.

19. Apro J, Tietge UJ, Dikkers A, Parini P, Angelin B, Rudling M. Impaired Cholesterol Efflux Capacity of High-Density Lipoprotein Isolated From Interstitial Fluid in Type 2 Diabetes Mellitus-Brief Report. Arterioscler Thromb Vasc Biol. 2016;36(5):787-91

20. Low Wang CC, Hess CN, Hiatt WR, Goldfine AB. Clinical Update: Cardiovascular Disease in Diabetes Mellitus: Atherosclerotic Cardiovascular Disease and Heart Failure in Type 2 Diabetes Mellitus - Mechanisms, Management, and Clinical Considerations. Circulation. 2016;133(24):2459-502.

21. Taskinen MR, Boren J. New insights into the pathophysiology of dyslipidemia in type 2 diabetes. Atherosclerosis. 2015;239(2):483-95.

22. Ginsberg HN, MacCallum PR. The obesity, metabolic syndrome, and type 2 diabetes mellitus pandemic: Part I. Increased cardiovascular disease risk and the importance of atherogenic dyslipidemia in persons with the metabolic syndrome and type 2 diabetes mellitus. J Cardiometab Syndr. 2009;4(2):113-9

23. Wu JY; Duan XY, Li L, Dai F, Li YY, Li XJ, et al. Dyslipidemia in Shanghai, China. Prev Med. 2010; 51:412-5.

24. Abd alamir M, Goyfman M, Chaus A, Dabbous F, Tamura L, Sandfort TV, et al. The Correlation of Dyslipidemia with the Extent of Coronary Artery Disease in the Multiethnic Study of Atherosclerosis. Journal of Lipids. 2018:1-9. 